\title{
MUCOUS FLOW AND CILIARY ACTIVITY IN THE TRACHEA OF RATS EXPOSED TO PULMONARY IRRITANT GAS*
}

\author{
BY \\ TORE DALHAMN and JOHANNES RHODIN \\ From the Department of Occupational Hygiene, the National Institute of Public Health, and the \\ Department of Anatomy, Karolinska Institute, Stockholm, Sweden
}

(RECEIVED FOR PUBLICATION JUNE 24, 1955)

In the field of occupational hygiene questions frequently arise concerning the effect on the respiratory tract of inhaled gases and of vapours from industrial solvents. The action of such agents is primarily on the mucosa of the respiratory tract.

Any study of this question necessarily involves determination of the rates of mucous flow and ciliary beat and investigations of possible morphological changes. The literature contains a few reports dealing with mucous flow and ciliary activity in various animals, e.g., by Ballenger (1949), Gray (1923), Lucas (1932), and Proetz (1933). Such studies, however, were not systematically carried out and did not pay sufficient attention to important physiological factors such as the temperature and moisture of the tissue examined. Moreover, the great majority of the experiments were performed in vitro, a procedure which several writers have strongly criticized (Barclay, Franklin, and Macbeth, 1937; Gordonoff and Mauderli, 1936).

A method devised by Dalhamn (1955), on which a preliminary report has been published, was used in the present study. This is part of a more extensive investigation of relevant questions, which it is intended to publish later in more complete form. For present purposes it is sufficient to say that in 37 healthy rats the mean rate of mucous flow in the trachea was found to be $13.5 \mathrm{~mm}$. per minute and the mean rate of ciliary activity 1,317 beats per minute.

In regard to ciliary movement, however, the frequency of beat is not the only factor of interest. In several reports in the literature it has been stated that the ciliary beat in many lower animals such as mussels and oysters consists of a rapid phase, the " effective stroke", and a slower " recovery stroke". The time relationship between the two was given as

* From a paper read before the Swedish Society of Physiologists on May 6, 1955.
$1: 2$ to $1: 5$. No study has hitherto been made of the corresponding time ratio in vertebrates.

Investigations of the phases of ciliary movement were made in the present experiments with a special high-speed cinematograph camera, permitting about 1,000 exposures per second. Thus each image was registered within a millisecond. Such rapid registration imposes very great demands on the film material and on the lighting arrangements. When the film is projected one sees, particularly at the edges of the light reflection, a rhythmic flaring up and diminution. From photomicrographs of some of the cinematograph films the time relationship between the rapid flare of the reflex and its gradual diminution was calculated to be approximately $1: 3$.

Rats were subjected to prolonged exposure to $\mathrm{SO}_{2}$ in the currently accepted maximum allowable concentration, i.e., 10 p.p.m. or $10^{-3}$ volumes $\%$. Nine rats were so exposed six hours daily for about 10 weeks. Six of the nine were then studied for secretory flow and rate of ciliary beat in the trachea. The three remaining animals were not exposed to $\mathrm{SO}_{2}$ during the next four weeks.

Immediately the trachea of the exposed rats was opened it was noted that the amount of secretion was greatly in excess of normal and that the mucosa was vascularized and reddened. Study of the mucous flow showed that this had ceased entirely in several cases and in the others was much slower than in normal rats. The mean rate of flow in the six rats was $5 \mathrm{~mm}$. per minute.

The frequency of ciliary beat, on the other hand, seemed unaffected, being similar to that in unexposed rats, i.e., a mean of 1,320 beats per minute.

On first thoughts it may appear contradictory that the secretory flow should slow in the presence of normal frequency of the ciliary beat. This, however, may be explained in several ways. Sjöstrand (1941) pointed out that one may expect 


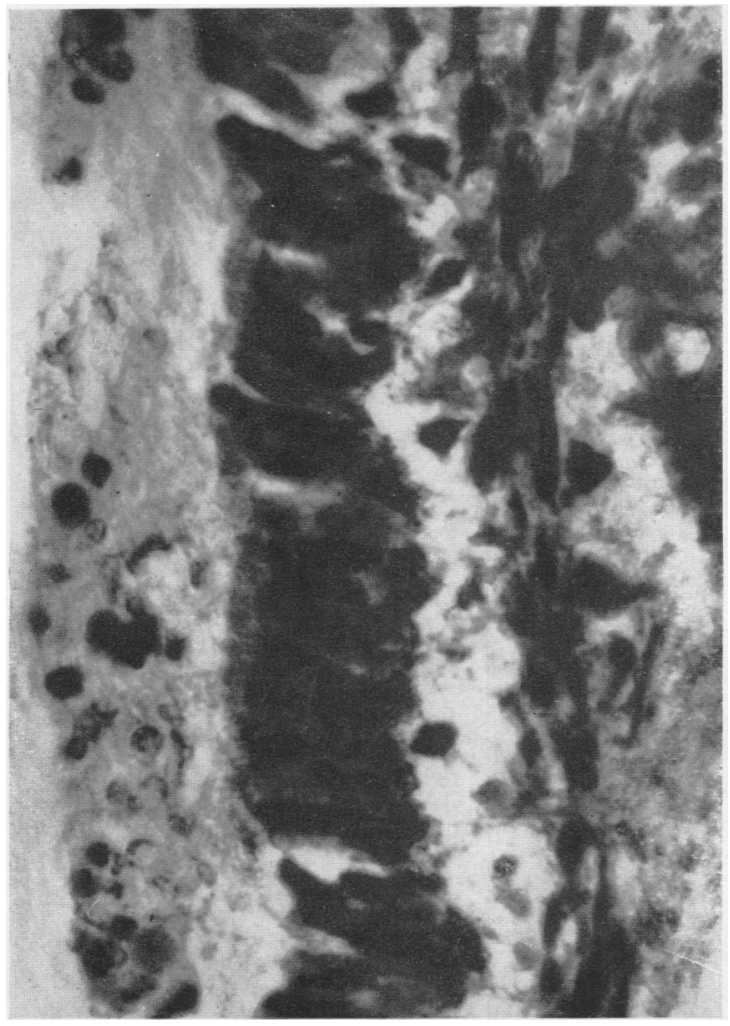

FIG. 1.-Freezed-fixed mucosa from, a rat exposed for 67 days to 11.5 p.p.m. SO. The mucous blanket is considerably thicker than normal. The secretion contains numerous cellular elements. $\times 830$.

an insignificant flow of mucus if the thickness of the secretory layer is four to five times as great as the length of the cilia. As stated above, the trachea of the exposed animals was immediately seen to contain a greatly increased amount of mucus. It was of interest, however, to obtain an objective measurement of this increase. By using the freeze-dried fixation method described by Sjöstrand (1944) it was possible to demonstrate that the mucosal layer in the trachea of the exposed rats was very much thickened, appeared more compact than normal, and was strewn with shed cells of various types (Fig. 1). In normal rats the thickness of the mucous layer was about $5 \mu$ and in the exposed rats about $25 \mu$.

Yet it is not only the thickening of the mucosa which may give rise to retardation of the secretory flow when the rate of ciliary beat remains unaltered. The viscosity of the secretion also plays a decisive part. Because of the anatomical and physiological conditions in the trachea of the rat it was found to be extremely difficult to obtain a reliable method for determining the viscosity of the tracheal secretion.

Still another factor which may contribute to slowing the mucous flow is a change in the time relationship of the ciliary phases, involving a levelling effect. Study of the movements of single cilia, however, failed to show any shift in this ratio. The reduced mucous flow in the exposed rats, therefore, would seem to have been primarily attributable to alterations in the viscosity or quantity of the secretion, or to a combination of both factors.

Analogous experiments, with the exception of study of the movement of ciliary phases, were made on the three rats which had been allowed to rest for four weeks following exposure to $\mathrm{SO}_{2}$. The results were in complete conformity with those in the rats examined directly after exposure. Thus there was still reduced mucous flow of the same order as in animals which had not been rested. The mucosa was reddened and the mucous layer much thicker than normal. Although this latter group was small, the findings in all three rats pointed clearly in the same direction.

The tracheal mucosa was studied after exposure to $\mathrm{SO}_{2}$ by microscopy and electron microscopy.

Microscopy, both immediately and four weeks after exposure to $\mathrm{SO}_{2}$, showed severe changes in the tracheal epithelium. This finding has already been reported (Dalhamn, 1955). Both the epithelium and the lamina propria were affected. The epithelium thus showed very long and slender ciliated cells and its surface, which normally is level and smooth, displayed in places very deep crypts. The cilia appeared to be very tightly packed on the surface of the cells.

In the lamina propria the most obvious change was severe oedema, on which the entire epithelium seemed to float freely. In addition there was profuse vascularization and perivascular extravasation of blood. In places the collagen fibrils were split and fragmented (Rhodin and Dalhamn, 1955).

Of the electron microscope studies only those concerning the structure of the cilia will be dealt with here, as these are of the greatest interest in the present connexion.

In a later report (Rhodin and Dalhamn, 1956) a detailed description will be given of the ciliary ultra-structure. The following paragraph contains a brief summary.

In its free portion a cilium consists of nine peripheral pairs of filaments, each pair with a common partition (Figs. 2 and 3 ). In the centre of the cilium are two distinctly separate filaments. The filaments are surrounded by a membrane 60 


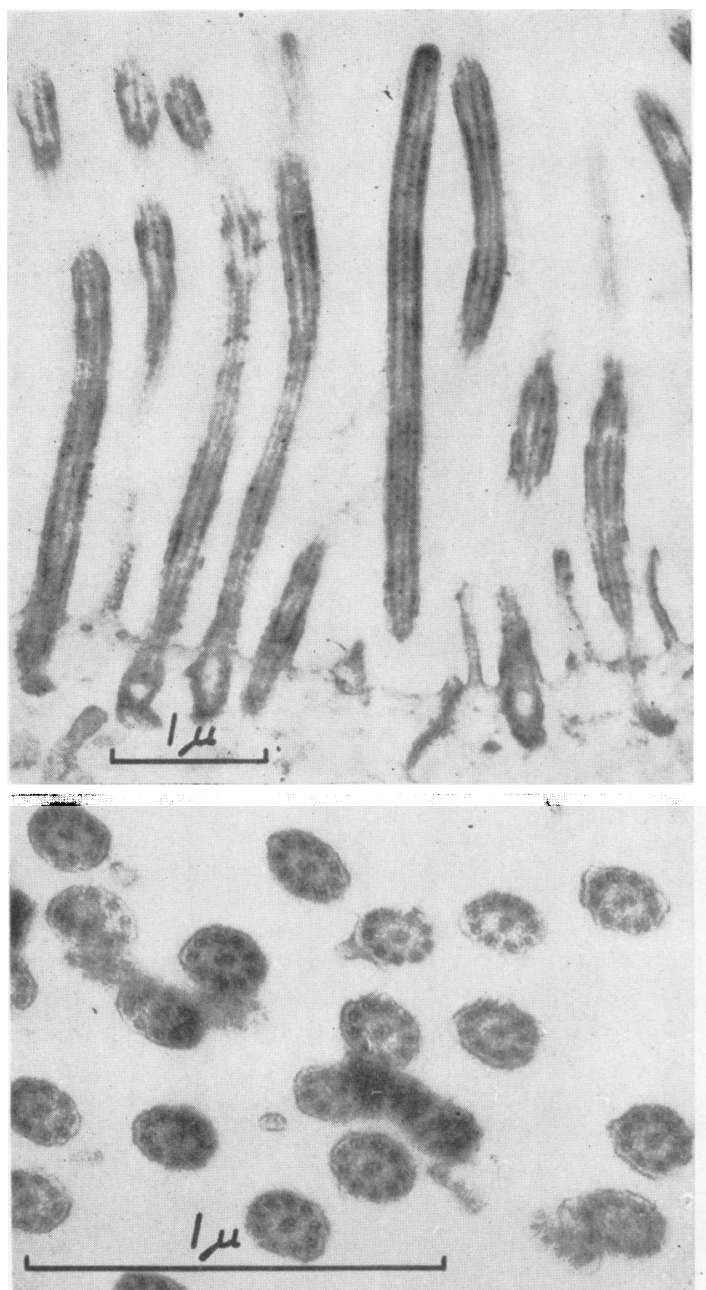

FIG. 2 (top, left).-Longitudinal section of cilia from the normal trachea of rat. $\times 15,750$.

Fig. 3 (below, left).-Cross section of cilia from the normal trachea of rat. $\times 42,750$.

Ångström units in thickness and thus appear as tubular structures. In longitudinal sections the peripheral filaments are seen to extend beyond the surface of the cell and down into the basal corpuscle, in which they are tightly packed around a weakly osmiophilic substance, in the centre of which can be observed a strongly osmiophilic body. The two central filaments, on the other hand, do not reach down into the basal corpuscle, but appear to terminate roughly on a level with the cell surface. In cross sections from the region of the basal corpuscles all the peripheral paired filaments are clearly visible in a garland-shaped arrangement. At the lower tip of the corpuscle may be observed a number of fine

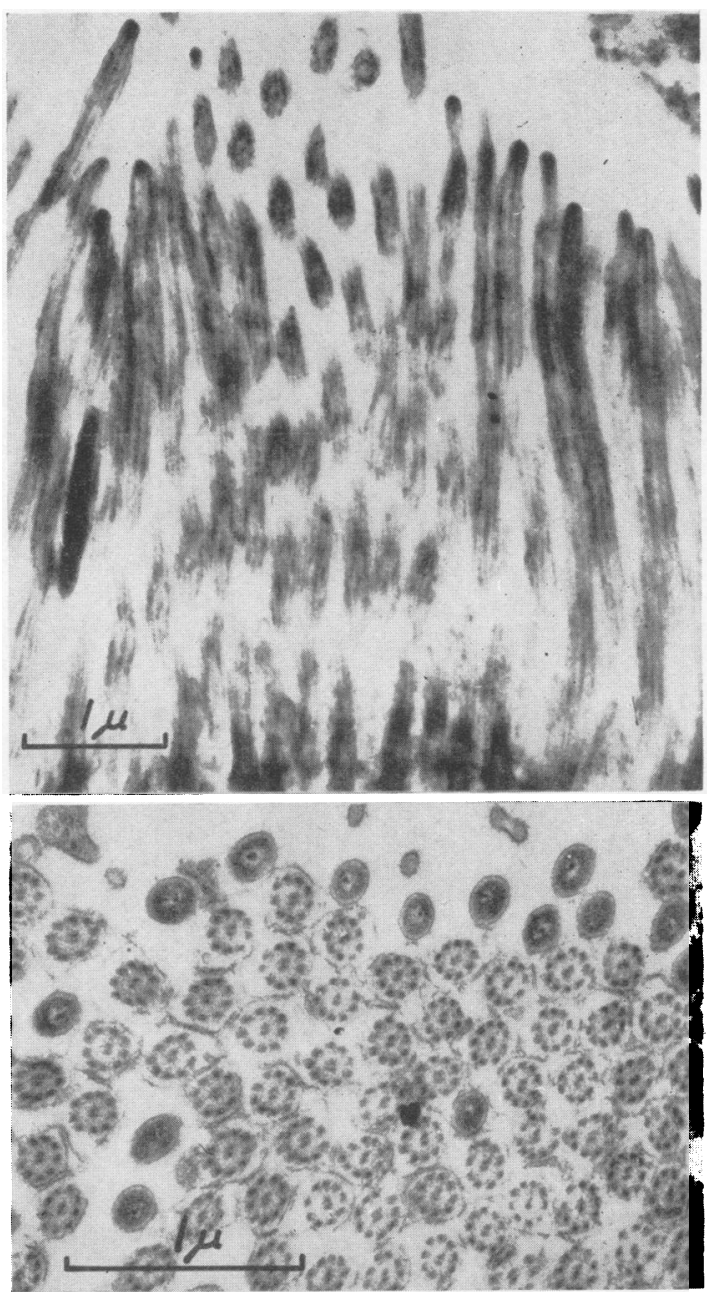

FIG. 4 (top, right).-Longitudinal section of closely packed cilia after exposure to $\mathrm{SO}_{2}$ for 10 weeks. $\times 13,250$.

FIG. 5 (below, right).-Cross section of cilia after exposure to $\mathrm{SO}_{2}$ for 10 weeks. Note the abundant dense bacteria. $\times 24,000$.

rootlet fibres which can be followed only for a short distance inwards towards the interior of the cell. Immediately below the basal corpuscle is an accumulation of oval or spherical mitochondria separated from the cytoplasm by a double membrane ; they also have an internal system of such membranes which, in contrast to earlier described types, are clearly branched.

Ultra-structural study of tracheal cilia in the rats exposed to $\mathrm{SO}_{2}$ revealed no evidence of changes in the free portion of the cilia, in the basal corpuscles, or in the mitochondria (Figs. 4 and 5). The increased density of the cilia noted at microscopy, on the other hand, was clearly discernible, and in all 
probability was caused by a rediction of the free surface of the ciliated cells.

\section{Results and Conclusions}

The apparatus employed permits meticulous determination of the rates of mucous flow and ciliary beat. With this method the action of certain pulmonary irritant gases has been studied. For the prolonged exposure herein discussed, $\mathrm{SO}_{2}$ was chosen. It has earlier been demonstrated (Amdur, Melvin, and Drinker, 1953) that even low concentrations of this gas may give rise to several changes in the respiratory pattern.

The results of the present experiments show that functional and morphological changes occurred after 10 weeks' exposure to 10 p.p.m. $\left(10^{-3}\right.$ volumes $\% \mathrm{SO}_{2}$. Thus the rate of mucous flow was considerably reduced and the amount of secretion greatly increased. The rate of ciliary beat, on the other hand, was unaffected. Nor did the phases of the ciliary beat appear to alter in character.

The morphological reactions were mainly seen in the epithelium and lamina propria. The epithelial cells were long and slender with com- pressed nuclei. The surface facing into the tracheal lumen was very irregular and showed deep crypts.

In the lamina propria there was severe oedema with splitting and fragmentation of the collagen fibrils, profuse vascularization, and blood escaping perivascularly.

The electron microscopic studies reported here particularly concerned ciliary structure. This did not differ from the structure observed in healthy rats.

It would thus seem that the functional changes noted are primarily attributable to alterations in the character of the mucosal secretion and not to injury to the ciliary mechanism as such. The results of the morphological studies confirmed these findings.

\section{REFERENCES}

Amdur, M. O., Melvin, W. W., and Drinker, P. (1953). Lancet, 2, 758 Ballenger, J. J.' (1949). Ann. Otol. (St. Louis), 58, 351.

Barclay, A. E., Franklin, K. J., and Macbeth, R. G. (1937) J. Physiol. (Lond.), 90, 347.

Gordonof, T., and Mauderli, H. (1936). Z. ges. exp. Med., 98, 265. Gray, J (1923) Proc, roy. Soc. B 95. 6 .

Lucas, A. M. (1932). J. Morph., 53, 265.

Proetz, A. W. (1933). Ann. Otol. (St. Louis), 42, 778.

Rhodin, J., and Dalhamn, T. (1955), Exp. Cell Res., 9, 371.

Rhodin, J., and Dalhamn, T. (1955). Exp. C

Sjöstrand, F. (1944). Acta anat. (Basel), 1, Suppl. 1.

Sjöstrand, T. (1941). Nord. Med. 10, 2023. 